\title{
Responses and Periodic Variations of Cosmic Ray Intensity and Solar Wind Speed to Sunspot Numbers
}

\author{
Jacob Oloketuyi $\mathbb{D}^{1,2,3}$ Yu Liu ${ }^{10,}{ }^{1,2}$ Amobichukwu Chukwudi Amanambu, ${ }^{4}$ \\ and Mingyu Zhao ${ }^{1,2}$ \\ ${ }^{1}$ Yunnan Observatories, Chinese Academy of Sciences, Kunming 650011, China \\ ${ }^{2}$ Shandong Provincial Key Laboratory of Optical Astronomy and Solar-Terrestrial Environment, Shandong University, \\ Weihai 264209, China \\ ${ }^{3}$ University of Chinese Academy of Sciences, Beijing 100049, China \\ ${ }^{4}$ Department of Geography, University of Florida, Gainesville, FL, USA
}

Correspondence should be addressed to Yu Liu; lyu@ynao.ac.cn

Received 4 November 2019; Revised 18 December 2019; Accepted 27 January 2020; Published 24 February 2020

Academic Editor: Geza Kovacs

Copyright (c) 2020 Jacob Oloketuyi et al. This is an open access article distributed under the Creative Commons Attribution License, which permits unrestricted use, distribution, and reproduction in any medium, provided the original work is properly cited.

\begin{abstract}
To investigate the periodic behaviour and relationship of sunspot numbers with cosmic ray intensity and solar wind speed, we present analysis from daily data generated from 1995 January to 2018 December. Cross-correlation and wavelet transform tools were employed to carry out the investigation. The analyses confirmed that the cosmic ray intensity correlates negatively with the sunspot numbers, exhibiting an asynchronous phase relationship with a strong negative correlation. The trend in cosmic ray intensity indicates that it undergoes the 11-year modulation that mainly depends on the solar activity in the heliosphere. On the other hand, the solar wind speed neither shows a clear phase relationship nor correlates with the sunspot numbers but shows a wide range of periodicities that could possibly be connected to the pattern of coronal hole configuration. A number of short and midterm variations were also observed from the wavelet analysis, i.e., 64-128 and 128-256 days for the cosmic ray intensity, 4-8, 32-64, 128-256, and 256-512 days for the solar wind speed, and 16-32, 32-64, 128-256, and 256-512 days for the sunspot numbers.
\end{abstract}

\section{Introduction}

It is well known that various of solar activities are closely associated with solar magnetic field processes and the study of the long-term evolution of solar activities are useful for the understanding of the solar atmosphere and the dynamo theories (e.g., [1-4]). The sunspot numbers (SSN), being the most important index of the solar activities, have been widely studied together with other indices [5-13].

Cosmic rays are highly energetic particles striking the earth from space. They can be originated from two primary sources: heliospheric and galactic cosmic rays (GCR). The solar wind is electrically charged, and the energised particles can move at the speed of about $400 \mathrm{~km} \cdot \mathrm{s}^{-1}$ freely in the heliospheric space. Both the cosmic ray intensity and the solar wind speed (SWS) are closely related to solar activity variations. They exhibit variations over different periods of time [11, 14-22].

Cosmic ray intensity (CRI) varies with numerous causes including the solar wind parameters and sunspots numbers. Mishra et al. [23] showed that solar wind velocity has a strong positive correlation with CRI during solar cycle 21. According to Jokipii and Thomas [24], variation in the observational angular parameter of heliographic equator could cause considerable changes in the galactic cosmic ray intensity. Long-term cosmic ray evolution can indicate solar cycle effect. Some attributes of cosmic rays and their behaviour in 11-year cycle with the interplanetary magnetic field (IMF) showed decrease in GCR flux that corresponds to an increase in the IMF intensity [25]. Ahluwalia [26] 


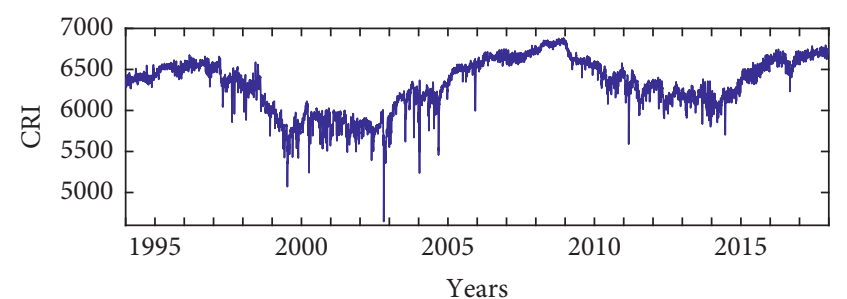

(a)

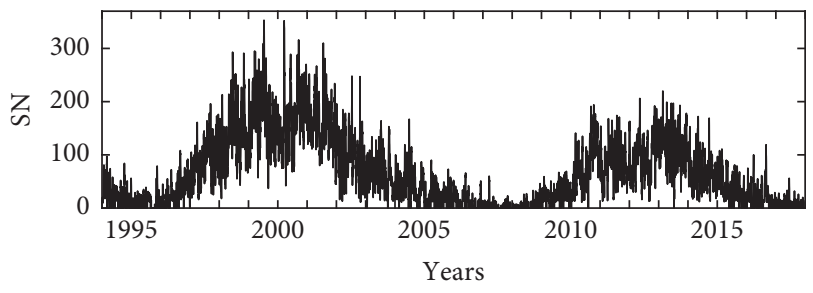

(c)

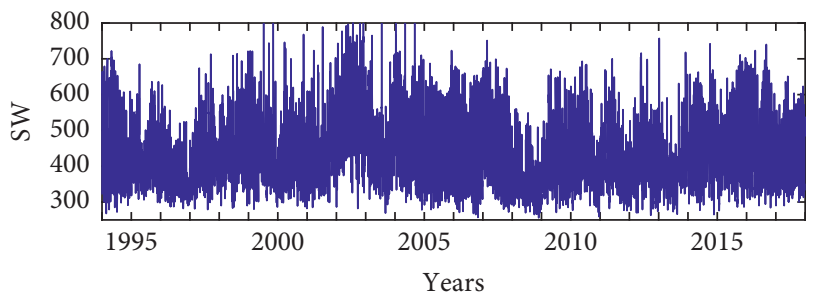

(e)

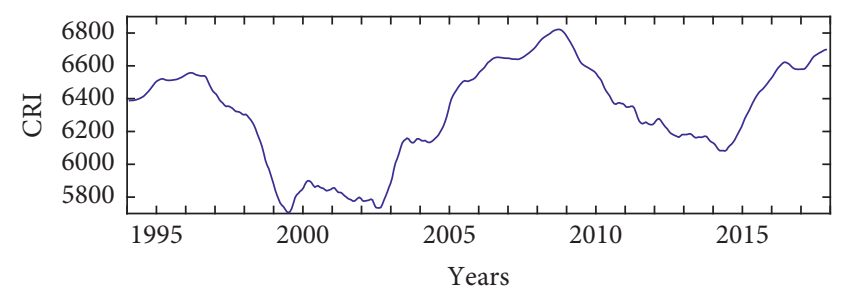

(b)

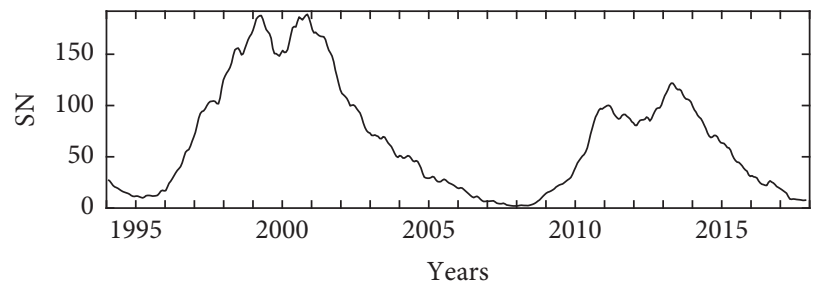

(d)

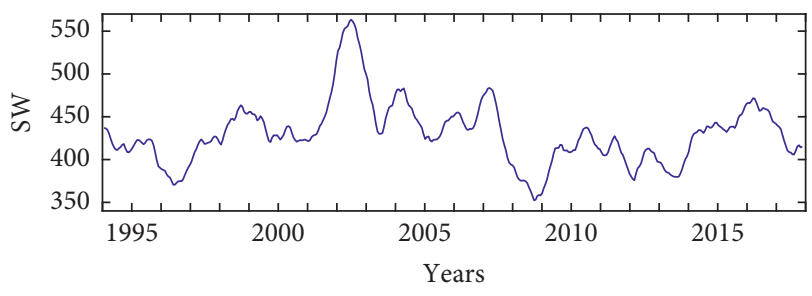

(f)

Figure 1: The daily distributions and the trends for the sunspot numbers, cosmic ray intensity, and the solar wind speed. The trends reveal the modulations and the long-term responses of the cosmic ray intensity and the solar wind speed to the sunspot numbers. The cosmic ray intensity shows periodicity around 11-year solar cycle while the solar wind speed shows irregular modulations in response to the solar activities. (a) Cosmic ray intensity daily distribution.(b) Cosmic ray intensity trend. (c) Sunspot numbers daily distribution. (d) Sunspot numbers trend. (e) Solar wind speed daily distribution. (f) Solar wind speed trend.

attributed the decrease in observed galactic cosmic ray flux to the increase in residual modulation within the heliosphere using GCR data from 1937 to 1998 . It was also noted that there was no increase in SSN observed during the same time period of observation. Fujimoto et al. [6] observed changes of CRI with solar activity; Hempelmann and Weber [27] used Fourier analysis of the time series and suspected that the cosmic rays have a link with solar activity following their observations that showed significant peaks of 10.7, 22.4, and 14.9 years.

Yan et al. [28] investigated the phase relationship between SSN, flare index, and solar radio flux using crosscorrelation analysis. Pérez-Peraza et al. [29] presented evidence of the existence of cosmic ray fluctuations with a periodicity of around 30 years. Li et al. [22] and Li et al. [30] investigated the periodicities in daily SWS and observed different periods in low and high wind speeds.

There have been many diverse studies carried out to understand several mechanisms in our solar system, which would make useful tools in the knowledge and understanding of our space weather and protection of artificial outer space objects. Oloketuyi et al. [31] investigated the influence of sunspot group numbers on the flare classes, finding that different class of flares respond differently to sunspot group emergencies. This study, however, investigated the behaviour and responses of CRI observed at the earth and SWS to the solar magnetic activity using SSN. The present study is an attempt to discover new periodicities and relationship between SSN and CRI with SWS that will broaden our understanding of cosmic ray modulations within the heliosphere in respect to the solar activities.

\section{Data and Methods}

2.1. Data. The data used in this study were obtained from different sources between 1995 January and 2018 December. The period of investigation covers solar cycles 23 to the present cycle 24 using daily data. The obtained data were used to investigate the periodic variation of SSN with CRI and SWS.

(1) Daily cosmic ray: the CRI data used in this study are pressure-corrected and obtained from the Cosmic Ray Station of the University of Oulu/Sodankyla Geophysical Observatory. The data are set to 1440 minutes auto resolution and can be downloaded from http://cosmicrays.oulu.fi/. Figure 1(a) shows the daily distributions of the CRI data.

(2) Daily sunspot numbers: the daily SSN used in this study was obtained from the Sunspot Index and Long-term Solar Observations. The World Data Center for the dissemination of international 
sunspot numbers is available at http://www.sidc.be/ silso/datafiles. The daily SSN distribution is presented in Figure 1(c).

(3) Daily solar wind: the daily SWS data used was generated by several spacecraft orbiting the Earth and can be obtained from https://spdf.gsfc.nasa.gov/pub/data/ omni/low_res_omni/omni_m_daily.dat. The daily distributions of SWS is presented in Figure 1(e).

2.2. Methods. To achieve the objective of detecting the responses and significant periodicities of CRI and the SWS to SSN in the investigated solar cycles, the cross-correlation analysis, continuous wavelet transforms, and the wavelet coherence methods were employed.

2.2.1. Cross-Correlation Analysis. The cross-correlation analysis (CCA) is a well-known method used to find where two signals match. Several authors have used this method. The coefficient of cross-correlation between the two data series is defined as [32]

$$
\operatorname{CC}(\Delta)=\frac{\sum_{i=1}^{n}[p(i)-\{p\}][q(i+\Delta)-\{q\}]}{(n-1) \delta_{p} \delta_{q}},
$$

in which $\{p\}$ represents the mean value of SSN, $\{q\}$ represents the mean value for CRI or SWS, $\delta_{p}$ and $\delta_{q}$ stand for their respective standard deviations. Positive coefficient $\Delta$ means that the time series of SSN leads the other parameters while negative coefficient means lag.

2.2.2. Wavelet Transform Methods. Wavelet analysis is a computational tool that helps in decompositions of varying signals into time and frequency dissections. This method helps in noise reduction from a signal by using the approximation process. The Continuous Wavelet Transform (CWT) has been noted to be a powerful tool for detecting the localised and quasiperiodic oscillations $[33,34]$. The wavelet coherence (WTC), an extended form of Continuous Wavelet Transform, is used in the analysis of time-frequency relationship between twotime varying signals [33, 35-39]. They reveal similarities in the states of two systems and allow the study of the synchronisation or phase difference in two-time series data [40].

The cross-wavelet transform (XWT) for the two-time varying signals SSN $\{x\}$ and CRI or SWS $\{y\}$ can be defined as

$$
X^{x y}=X^{x} X^{y *} .
$$

The $X^{x}$ and $X^{y}$ designate the continuous wavelet transform of the time-varying signals SSN $\{x\}$ and CRI or SWS $\{y\}$. The $*$ designates complex conjugation. The complex argument $\arg X^{x y}$ can be considered as a localised relative phase between the SSN $\{x\}$ and CRI or SWS $\{y\}$ in the time-frequency domain which is the phase angle difference between them [33].
The continuous wavelet transforms of a continuous function $x(t)$ relative to a real-valued wavelet $\psi(t)$ is described by [41] as

$$
W_{\psi}(s, \tau)=\int_{-\infty}^{+\infty} x(t) \psi_{s, \tau}^{*}(t) \mathrm{d} t,
$$

where

$$
\psi_{s, \tau}(t)=\frac{1}{\sqrt{s}} \psi\left(\frac{t-\tau}{s}\right),
$$

in which $s$ and $\tau$ are called scale and translation parameters, respectively. $W_{\psi}(s, \tau)$, represents the wavelet transform coefficients and $\psi$ is the fundamental mother wavelet.

Wavelet Coherence (WTC) functions as a correlation coefficient, whereby the areas of high common power between two-time varying data are revealed in their time and frequency domains. This technique is unique and useful in the computation of time and frequency of signals such that it distinguishes significant coherence even in their common low power [33]. The importance of WTC is due to the circumstance that wavelet cross-spectrum looks not good for testing of the interrelation between two progressions $[42,43]$. The wavelet coherence of two time-varying signals $\mathrm{A}$ and $\mathrm{B}$ can be defined as

$$
R_{n}^{2}(s)=\frac{\left|S\left(s^{-1} X_{n}^{A B}(s)\right)\right|^{2}}{S\left(s^{-1}\left|X_{n}^{A}(s)\right|^{2}\right) * S\left(s^{-1}\left|X_{n}^{B}(s)\right|^{2}\right)},
$$

in which $S$ represents smoothing operator in both time and frequency components, $X^{A}$ represents the wavelets for the time-varying data of the SSN and $X^{B}$ for CRI or SWS. $X^{A B}$ is the cross wavelet. The cone of influence (COI) defines the wavelet power for a discontinuity at the edge decreases by a factor $e^{-2}$ [33]. The wavelet analysis was employed on all the data sets separately for the selected solar cycles and the combined solar cycles for all solar activity indices used.

The Morlet Wavelet function is represented by

$$
\psi_{0}(\eta)=\pi^{-1 / 4} e^{i \omega_{0} \eta} e^{-\eta^{2} / 2} .
$$

A plane sine wave with amplitude derived in time from the Gaussian function where $\omega_{0}$ is a nondimensional frequency. $\omega_{0}=6$ was adopted to give a good balance of spectral and temporal resolution $[33,36]$. For this study, 95 percent confidence level has been applied in the analysis [33].

\section{Results and Discussions}

Table 1 shows the correlation coefficients obtained between the daily SSN with CRI and SWS. The analysis shows that CRI is negatively correlated with SSN for the two solar cycles. The cross-correlation analysis shows strong anticorrelations with coefficients of -0.72 and -0.73 for cycles 23 and 24, respectively. However, the analysis for SWS with SSN shows inconsequential positive correlations.

Figure 1 shows the distributions and trends for SSN, CRI, and SWS. The trends were calculated using a 13-day moving average. From this figure, a significant increase in CRI was observed during the solar minima while decreasing at the 
TABLE 1: The correlation coefficients between the time series of sunspot numbers with cosmic ray intensity and solar wind speed.

\begin{tabular}{lccc}
\hline & Cycle 23 & Cycle 24 & Cycle 23+24 \\
& SSN & SSN & SSN \\
\hline Cosmic ray intensity & -0.72 & -0.73 & -0.73 \\
Significance & High & High & High \\
Solar wind speed & 0.05 & 0.02 & 0.06 \\
Significance & NC $^{*}$ & NC $^{*}$ & NC $^{*}$ \\
\hline
\end{tabular}

$\mathrm{NC}^{*}$ means No correlation.

peaks of the solar cycles. The trends perfectly reveal the variations they exhibit in long-term responses to SSN. The CRI shows periodicity similar to the 11-year solar cycle in the opposite while the SWS shows irregular modulations in response to the solar activities. This observation in CRI could be attributed to the varying amplitude of the heliospheric magnetic field at the solar maximum reducing the influx of galactic cosmic rays entering the solar system and vice versa at solar minima due to the low solar magnetic activity. This shows that CRI is free and constantly abundant outside the heliosphere, but its influence and modulations in the heliosphere are solely dependent on solar activities.

Figure 2 shows the cross-correlation analyses between daily SSN and daily CRI and SWS. The abscissa in each frame shows the lag or time shift with respect to SSN. The negative values designate backward shifts or lag, and if positive, it designates otherwise. The vertical dash line in each frame indicates no shift or phase boundary. From the cross-correlation analysis in Figure 2(a), CRI shows a strong negative correlation with SSN for the solar cycle 23. It lags behind SSN with coefficients of -0.72 . Moreover, CRI also shows anticorrelation with SSN with a coefficient of -0.73 in cycle 24 as shown in Figure 2(b). The two frames of Figures 2(c) and 2(d) show that SSN and SWS are uncorrelated in cycles 23 and 24 with coefficients of 0.05 and 0.02 , respectively.

Figure 3 shows the correlation analysis for the overall period. The analysis shows the overall behaviour of CRI and SWS with respect to SSN. The time series of CRI lags behind SSN at a correlation coefficient of -0.73 shown in Figure 3(a), while SWS is uncorrelated with SSN with a weak coefficient of 0.06 as shown in Figure 3(b).

\subsection{Wavelet Analysis}

3.1.1. Morlet Continuous Wavelet Transform. To study the periodic variations of the daily SSN with CRI and SWS, the Morlet wavelet transform was employed with the red noise approximation on the whole data used. The results are presented in Figures 4-6, where in each figure (a) shows the scale-average time series of daily data, (b) shows the wavelet power spectrum (WPS), and (c) shows their global wavelet spectrum (GWS). From the figures, the colours reveal the levels of power and the frequency in the wavelet power spectrum. The blue areas designate regions of low power while the yellow and the black areas designate regions of more substantial power. The thin black line in each panel (b) is the cone of influence (COI) which represents the 95 percent confidence level. This was introduced to minimise errors in the areas liable to have edge effects due to discontinuity, and it is padded with zeros. The GWS results, representing the power variation with period, are presented at panels (c).

Figure 4 shows the wavelet analysis for the daily CRI. The analysis shows periodicities of 64-128 and 128-256 days. The most prominent periodicity is $128-256$ days. This periodicity appeared in both solar cycles with a peak value of $2.47 \times 10^{6}$ on the global wavelet spectrum. The wavelet spectrum also shows that CRI is more dominant in cycle 23 with more variations and larger amplitudes.

The wavelet analysis for the daily SWS is presented in Figure 5. A wide range of periodicities were observed. The most significant periodicity is $256-512$ days which appeared in both cycles and peaked at $7.53 \times 10^{5}$ on the global wavelet spectrum. Periodicity of 4-8 days is also significant with $6.3 \times 10^{4}$ on the global wavelet spectrum analysis. Other noticeable periodicities include 32-64 days in 1996, 1999-2001, 2003-2005, and 2017, while periodicity of 128-256 days was observed in both cycles. For the SWS wavelet spectrum, both cycles seem to be moderately substantial in occurrence. The scale-average time series shows SWS has its higher amplitudes at the descending phase of cycle 23.

Figure 6 shows the periodicities for the daily SSN. From this figure, SSN exhibit a wide range of periodicities in cycle 23. The observed periodicities include 16-32, 32-64, 128-256, and 256-512 days. Most of these periodicities appeared briefly in solar cycle 23 . The $16-32$ days periodicity appeared briefly in cycle 23 from 1999 to 2002 while 32-64 was observed briefly in both cycles 23 and 24 . The periodicity was observed shortly between 1999 and 2000 in cycle 23 and from late 2010 to early 2014 in cycle 24 . The most significant periodicity is 256 days which appeared in both solar cycles. The wavelet spectrum for SSN shows that solar cycle 24 is weaker compared to cycle 23. The amplitude of SSN is much higher during the solar cycle 23.

3.1.2. Wavelet Coherence (WTC). This study also employed the wavelet coherence method, an extended tool of wavelet transforms. The wavelet coherence analysis was used to examine the phase relationships CRI and SWS independently have with SSN. The results from the analyses are shown in Figure 7. The confidence level of wavelet coherence analysis presented is above 95 percent.

Figure 7(a) shows the WTC analysis for SSN and CRI while Figure 7(b) for SSN and SWS. The directions of the arrows indicate relative phase relationships existing between them. If the arrows point towards right-hand (left-hand), they indicate that SSN is in phase (antiphase) with CRI or SWS. When the arrows are pointing upward (downward), they indicate that the phase relationship is leading (lagging). The white dash line in each panel is the cone of influence (COI) introduced.

In Figure 7(a), SSN shows an asynchronous phase relationship with CRI. The yellow regions indicate a strong anticorrelation or antiphase relation at higher periodicities between 64 and 256 days in both cycles. The blue regions 


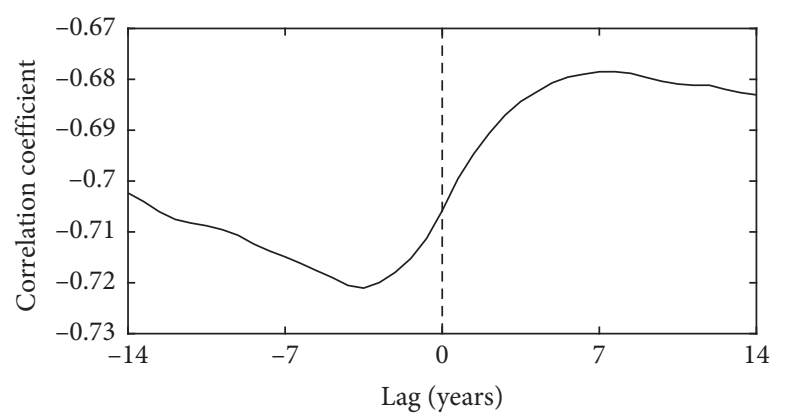

(a)

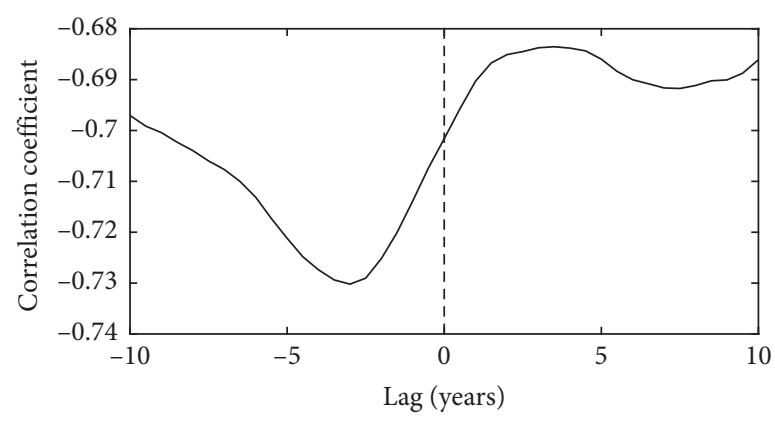

(c)

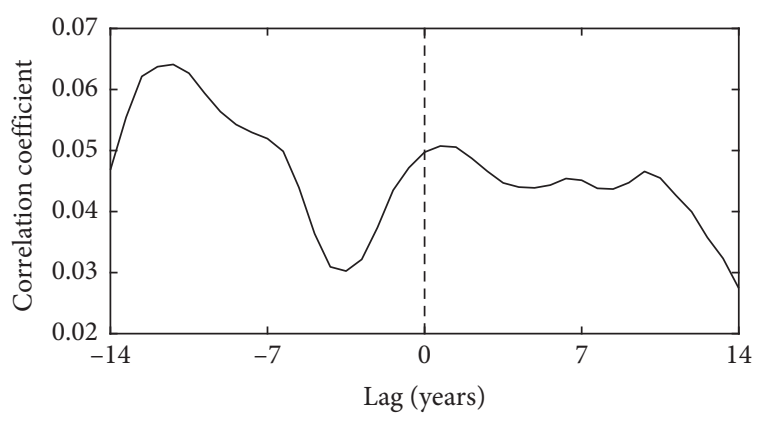

(b)

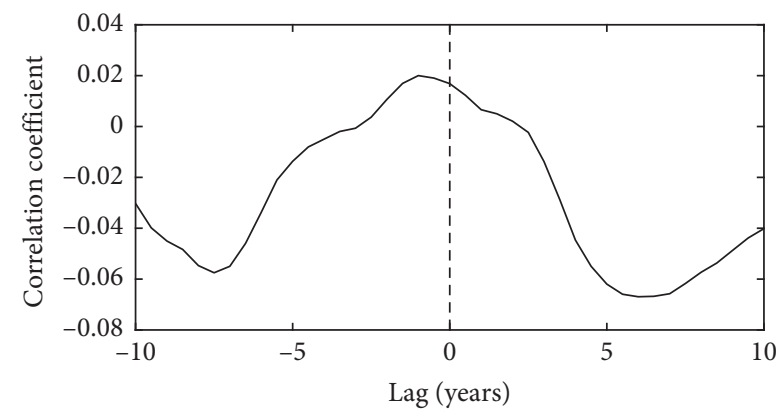

(d)

FIgURE 2: Cross-correlation coefficients of daily SSN-CRI and SSN-SWS for the two solar cycles 23 and 24, separately. The abscissa designates the time delay with respect to the daily sunspot numbers. (a) SSN-CRI solar cycle 23. (b) SSN-SWS solar cycle 23. (c) SSN-CRI solar cycle 24. (d) SSN-SWS solar cycle 24 .

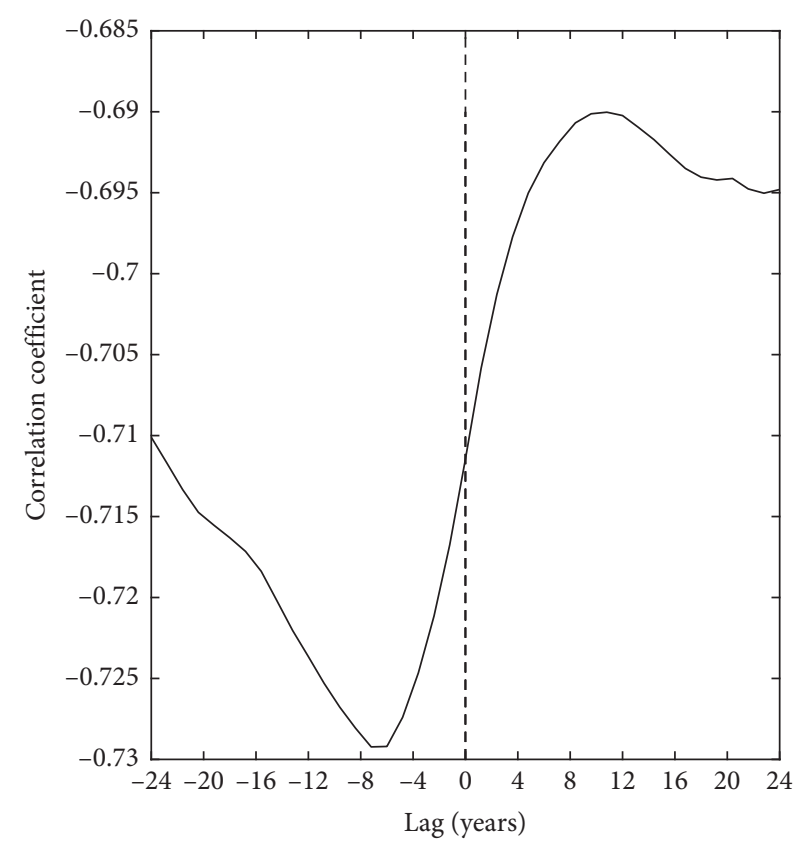

(a)

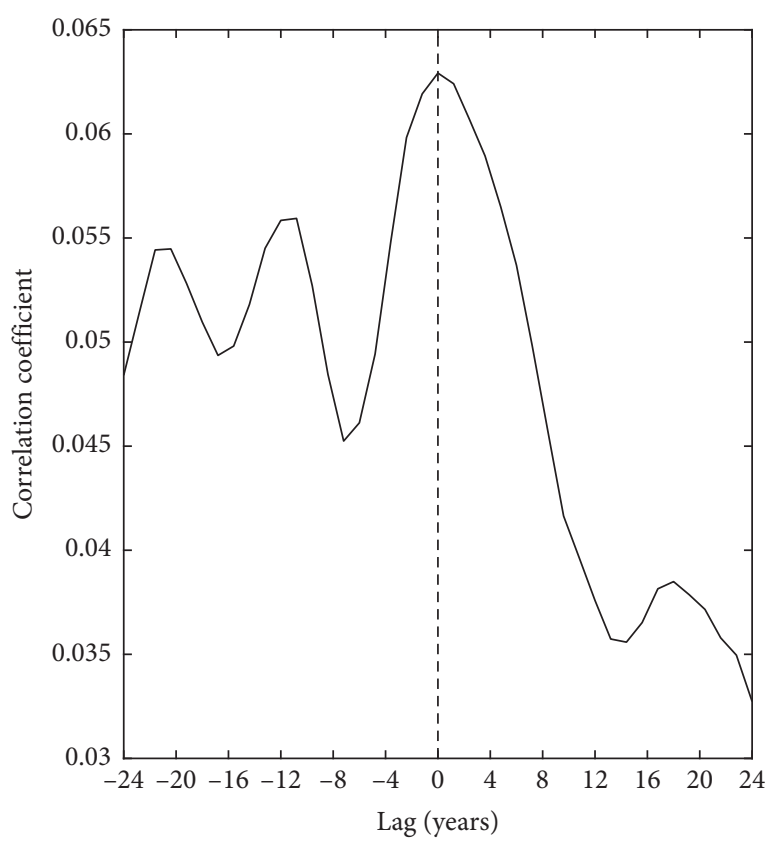

(b)

FIgURE 3: Cross-correlation coefficients of daily SSN-CRI and SSN-SWS for the two Solar Cycles 23 and 24 combined. The abscissa designates the time delay with respect to the daily sunspot numbers. (a) SSN-CRI. (b) SSN-SWS.

designate a low presence of phase interactions. The analysis shows that sunspot numbers leads the cosmic ray intensity and there is a slight phase coherence at periodicity around 8 days at the descending phase of cycle 23 from 2004 to 2006.
However, there is no other significant phase synchronisation at any point in the analysis. This could be attributed to their different and independent sources in the interplanetary space. 


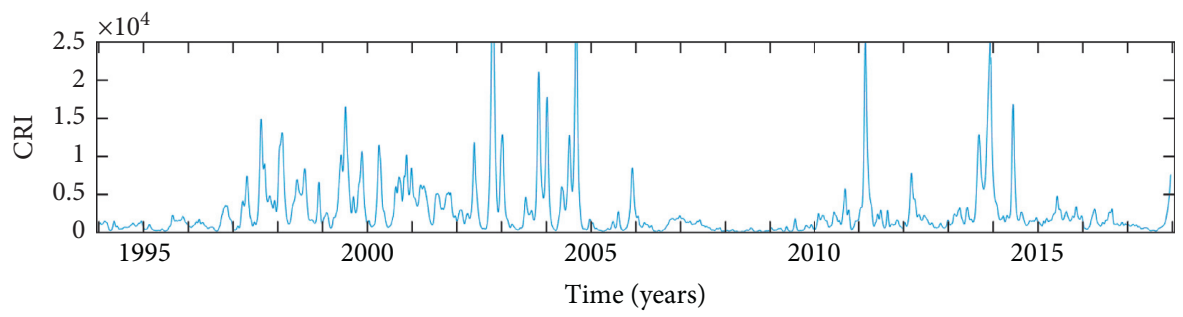

(a)

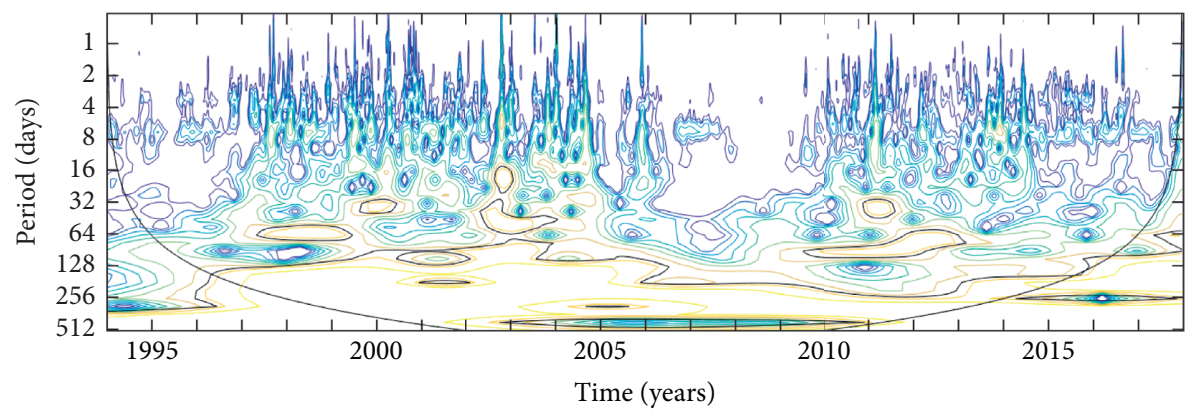

(b)

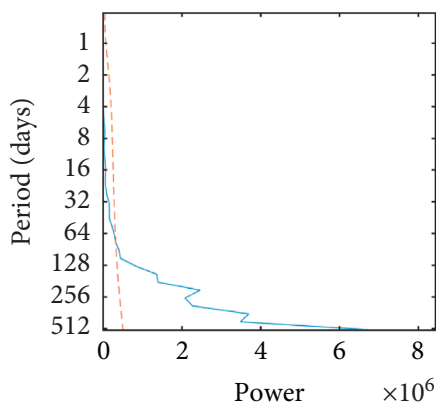

(c)

Figure 4: (a) The scale-average time series of daily cosmic ray intensity, (b) the wavelet spectrum, and (c) the global wavelet spectrum. For more details see the context.

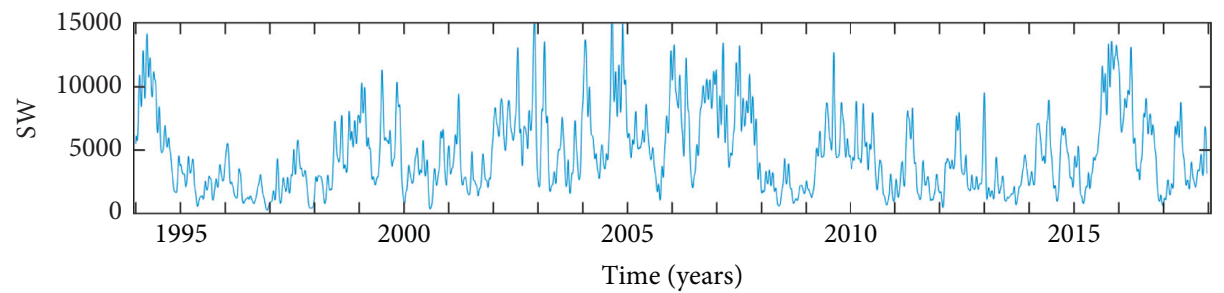

(a)

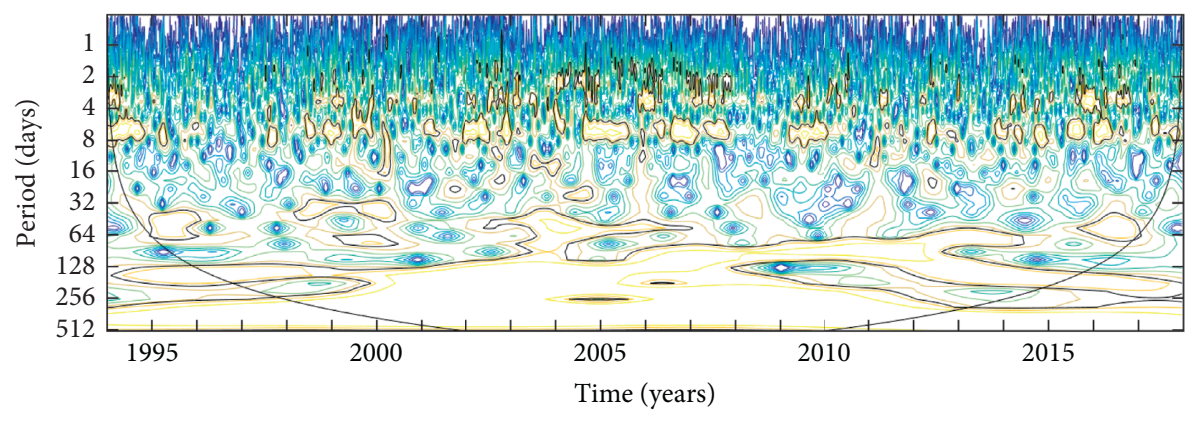

(b)

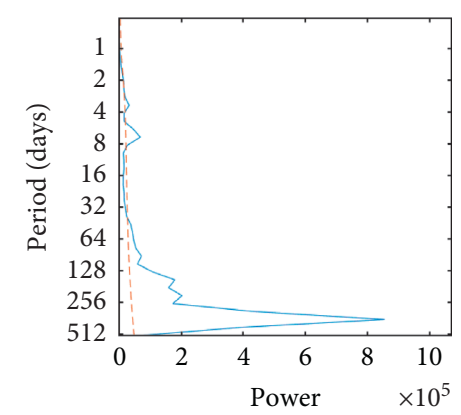

(c)

Figure 5: (a) The scale-average time series of daily solar wind speed, (b) the wavelet spectrum, and (c) the global wavelet spectrum.

Figure 7(b) shows the wavelet coherence analysis for SSN and SWS. The analysis shows no strong phase interaction across both solar cycles. However, phase coherence was relatively present occurring at some periodicities. The observed phase synchronisation appeared briefly from 2012 to 2015 and then 2017 at periodicity around 2 and 8 days. Other noticeable phase synchronisations observed include between 2- and 8-day periodicities from 2001 to 2005 and 1- and 2day variations from 2010 to 2012. SSN leads when the arrows are pointing eastward in yellow. The phase anticorrelations observed were mostly around descending and ascending phases of the solar cycles. Largely, the uneven phase interactions at different periodicities could be ascribed to the pattern of configuration in the coronal hole and the solar wind outflow from different sources due to the solar magnetic structures $[44,45]$.

The cross-correlation analysis agrees with previous studies. Forbush [46] and [47] confirmed the anticorrelation of CRI with SSN using data from different stations between 1937 and 1952. Mishra [48] used monthly data of Grouped 


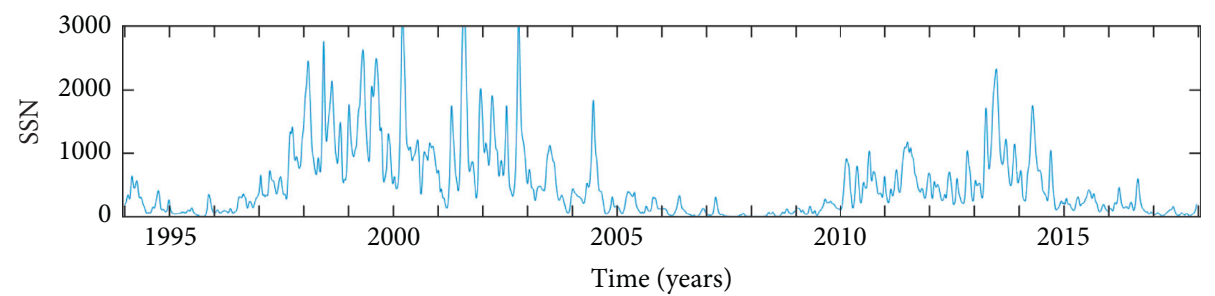

(a)

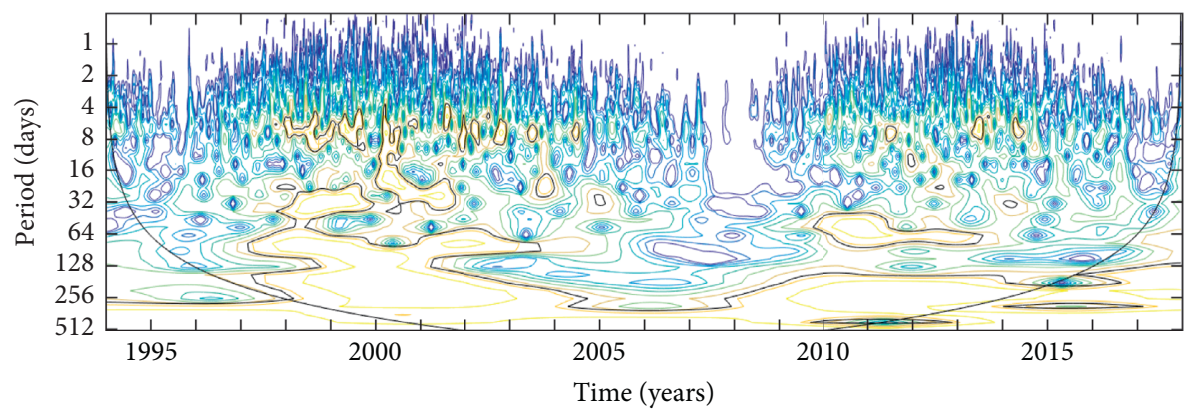

(b)

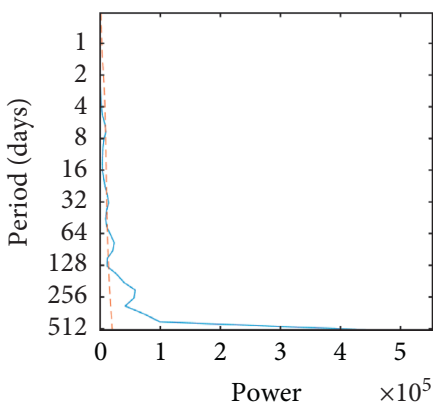

(c)

FIgURE 6: (a) The scale-average time series of daily sunspot numbers, (b) the wavelet spectrum, and (c) the global wavelet spectrum.

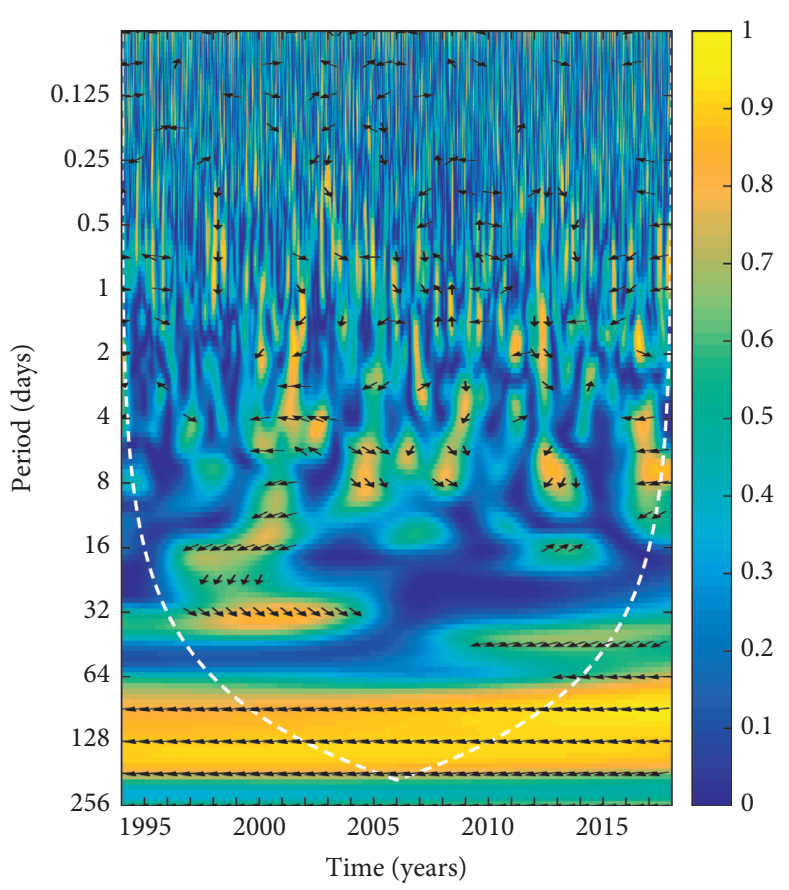

(a)

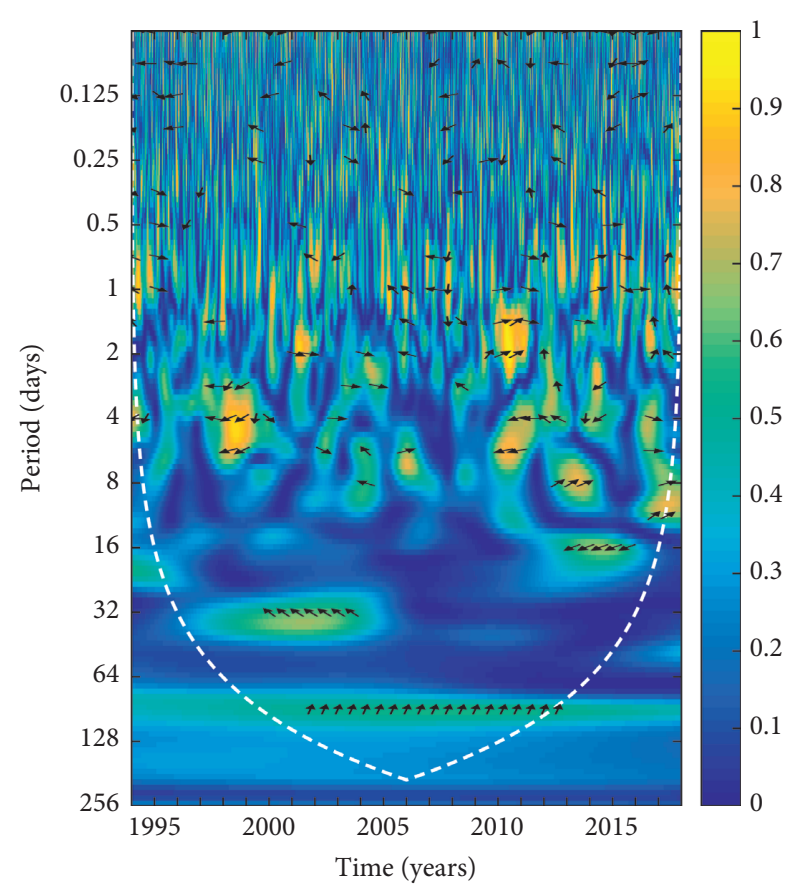

(b)

FIGURE 7: The wavelet coherence analysis for between sunspot numbers and cosmic ray intensity (a) and for between sunspot numbers and solar wind speed (b). For more details, see the context.

Solar Flare Index (GSF), instead of SSN and CRI for solar cycles 20 to 23 . It was observed that CRI negatively correlated with solar activities in cycles 20 to 23. Kane [15] and [11] also found that CRI is anticorrelated with SSN. However, Tiwari et al. [49] suggested that the strength of IMF characterised quantitive effect on the cosmic ray.
For the SWS Emery et al. [50] investigated different components of SWS, and periodicities of 9, 13.5, 27 days were observed. Other periodicities obtained include $120-145,145-165,270-300$ days and 1.3-1.4-year from the analysis. Singh and Gautam [51] studied solar wind parameters, and in the interplanetary magnetic field (IMF), a 
number of variations were found: $\sim 9, \sim 14, \sim 75, \sim 99, \sim 200$ days, $\sim 1.4$ years, $\sim 1.7$ years. These periodicities were observed in annual, semiannual, and triannual variations.

Sunspots are formed as a result of the sun's strong magnetic activities. These activities of solar magnetic field can lead to the heating of solar corona resulting in the escape of solar wind from the coronal hole. It was noted that the differences in SWS variations could be attributed to their different sources and configuration of coronal hole. Dunzlaff et al. [52] and Lario and Roelof [53] suggested that different coronal hole structures could lead to different co-rotating interaction region structures which could possibly also be applicable.

Joshi et al. [54] showed periodicities of $(\sim 175,133,113$, 104, 84, 63 days) in sunspot activity of solar cycle 23. Mendoza and Velasco-Herrera [55] showed the wavelet analysis of all the sunspot groups. The significant periodicities in the global spectrum are about $2,1.3,0.7,0.4$, and 0.20 years, the most prominent ones being 2 and 1.3 years. Their wavelet spectrum indicates that for cycles 16 to 19 and 21 and 22 the midterm periodicities (MTPs) are dominant. Chowdhury et al. [56] used power spectral and continuous wavelet analysis to investigate sunspot periodicities; in cycle 22 , a wide range of significant quasiperiodicities of 87-106, $159-175,194-219,292-318$, and 389 days were observed while in cycle 23 periods of $69-95,113-133,160-187$, $245-321,348-406 \mathrm{~d}$, and $\sim 1.3$ years were observed. The combined solar cycles 22 and 23 , periodicities of $24-43$, $50-73,86-120,130-180,240-270$ days and quasi-annual period of 330-380 days were observed. Yin et al. [57] also found periodicities between 23 and 36 days in solar cycle 15, 21-34 days in cycle 16, 24-35 days in cycle 18, and 23-33 days in cycles 20 and 22. Kilcik et al. [58] observed a periodicity of 300-370 days appeared only in cycles 18,20 , and 23. Oloketuyi et al. [31] found that the B flares responded differently from the other flares to sunspot group numbers by having cycles around 5 years of variation.

\section{Conclusions}

We have investigated the temporal and periodic variations of CRI, and SWS with SSN in the solar cycles 23 and 24 using cross-correlation and wavelet transforms. The results obtained are summarized as follows.

We found that CRI undergoes 11-year solar cycle within the heliosphere, which is greatly influenced mainly by solar activities. The cycle formed has its peak at the solar minimum and vice-versa. The present study also confirmed that the daily sunspot numbers and CRI are negatively correlated. The anticorrelations observed from the cycles are highly significant. SWS was found to be uncorrelated with SSN.

The wavelet analyses show a wide range of periodicities. The observed periodicities for the daily CRI include 64-128 and 128-256 days. The most prominent periodicity is 128-256 days which appeared in both cycles analysed. The observed daily SWS exhibits a wide range of periodicities. The obtained periodicities include 4-8, 32-64, 128-256, and 256-512 days which appeared in both cycles and the most significant periodicity with a peak value of $7.53 \times 10^{5}$ on the global wavelet spectrum. The wavelet analysis also shows that most of the periodicities for the daily sunspot numbers were obtained in solar cycle 23 . The obtained periodicities mostly appeared briefly include $16-32,32-64$, 128-256, and 256-512 days. The most significant periodicity is 256 days which appeared in both solar cycles. The wavelet spectrum shows solar activity cycle 24 is weaker compared to cycle 23 .

Recently, Singh [59] studied the short-term variations of SWS, CRI, interplanetary magnetic field, SWS, solar radio flux, and geomagnetic Ap Index. The study investigated solar cycles 20 to 24 covering the polarity reversal period and found periodicities of $2.5,4.5,9,14.5$, and 30.1 days for the SWS while the CRI 2.8, 13.1- and 18.5-day periodicities. Significant observed periodicities obtained for other indices include 18.5, 16.7, 13.5, 10.6, 9.1, 6.8, 5.5, 4.2, 2.8, 2.4, 1.8 days periods. Similarly, Tsichla et al. [60] investigated five solar cycles from 1965 to 2018 for new periodicities for the CRI, sunspots, Bz-component of the interplanetary magnetic field, and geomagnetic Ap Index. Periodicities observed are 5.5-, 6-, and 9-year and 13.9-day periods, including the 27-day, 11-year periods, and the 1.7- and 2.9year periodicities which are found to be integral multiples of the Rieger and QBO periods, respectively.

The analysis from the wavelet coherence also confirms that CRI and SSN correlate negatively. The analysis also shows that CRI lagged behind sunspot numbers. Largely, they exhibit an asynchronous phase relationship, a clear indication that CRI in the interplanetary space responds negatively to solar activity. On the other hand, SWS does not show a clear phase relationship with SSN but largely irregular phase interaction, a phenomenon that could be best described as noise, which could be connected to irregularities in coronal holes configurations where the high-speed solar wind originates from. However, the availability of a physically meaningful phase definition depends crucially on the appropriate choice of the reference frequency [61]. If we want to investigate the phase relationship between different solar activity indicators, we should be careful in choosing the reference periodic scales [62]. The low-frequency modes can be considered as a long-term trend and the high-frequency modes as a stochastic component that is not random but amplitude modulated [63].

The anticorrelation relationship of SSN with the CRI could be attributed largely to the influx of galactic cosmic ray into the heliospheric space, and the response of CRI shows that it could be useful in investigating the solar activities and other parameters like solar flares and the CMEs in the solar system. However, there is a need to make further investigation on solar magnetism and its mechanisms which are the primary source and causes of solar-related phenomena.

\section{Data Availability}

The authors express their appreciations to the providers of data used: Oulu Station for the cosmic ray data http:// cosmicrays.oulu.fi/, Sodankyla Geophysical Observatory, and the World Data Center for both solar wind (SWS: 
https://spdf.gsfc.nasa.gov/pub/data/omni/low_res_omni/ omni_m_daily.dat) and sunspot data (SSN: http:// www.sidc.be/silso/datafiles) and to the wavelet software provided by C. Torrence and G. Compo, http://paos. colorado.edu/research/wavelets/.

\section{Conflicts of Interest}

The authors declare that they have no conflicts of interest.

\section{Acknowledgments}

This work was funded by the grants from the Strategic Priority Research Program of CAS with grant XDA17040507, the CAS-TWAS President's Fellowship, and the National Science Foundation of China (NSFC 11533009, 11973086 , and 11603074). This work was also funded by the Project Supported by the Specialized Research Fund for Shandong Provincial Key Laboratory. In addition, the authors are also grateful to the One Belt and One Road Scientific Project of the West Light Foundation, CAS.

\section{References}

[1] Y. Shen, Y. Liu, J. Su, and Y. Deng, "On a coronal blowout jet: the first observation of a simultaneously produced bubble-like CME and a jet-like CME in a solar event," The Astrophysical Journal, vol. 745, no. 2, p. 164, 2012.

[2] H. Li, Y. Liu, and K. V. Tam, "Fundamental and harmonic oscillations in neighboring coronal loops," The Astrophysical Journal, vol. 842, no. 2, p. 99, 2017.

[3] Y. Miao, Y. Liu, H. B. Li et al., "A blowout jet associated with one obvious extreme-ultraviolet wave and one complicated coronal mass ejection event," The Astrophysical Journal, vol. 869, no. 1, p. 39, 2018.

[4] Y. Shen, Z. Tang, H. Li, and Y. Liu, "Coronal EUV, QFP, and kink waves simultaneously launched during the course of jetloop interaction," Monthly Notices of the Royal Astronomical Society: Letters, vol. 480, no. 1, pp. L63-L67, 2018.

[5] S. H. Schwabe, "Die sonne," Astronomische Nachrichten, vol. 20, no. 17, pp. 283-286, 1843

[6] K. Fujimoto, H. Kojima, and K. Murakami, "The Solar wind effect on cosmic rays and the solar activity," in Proceedings of the 19th International Cosmic Ray Conference (ICRC19), La Jolla, CA, USA, August 1985.

[7] N. A. Krivova and S. K. Solanki, "The 1.3-year and 156-day periodicities in sunspot data: wavelet analysis suggests a common origin," Astronomy \& Astrophysics, vol. 394, no. 2, pp. 701-706, 2002.

[8] J. King and N. Papitashvili, "Solar wind spatial scales in and comparisons of hourly Wind and ACE plasma and magnetic field data," Journal of Geophysical Research: Space Physics, vol. 110, no. A2, 2005.

[9] S. P. Nayar, "Periodicities in solar activity and their signature in the terrestrial environment," in Proceedings of the ILWS Workshop "the Solar Influence on the Heliosphere and Earth's Environment: Recent Progress and Prospects", Goa, India, February 2006.

[10] H. Kojima, Y. Hayashi, K. Hayashi et al., "The change in cosmic ray intensity variation with the solar wind velocity (using GRAPES-3 muon narrow angle telescopes and kiel neutron monitor)," in Proceedings of the International Cosmic Ray Conference, p. 557, Yucatan, Mexico, July 2008.

[11] R. P. Kane, "Hysteresis of cosmic rays with respect to sunspot numbers during the recent sunspot minimum," Solar Physics, vol. 269, no. 2, pp. 451-454, 2011.

[12] R. Singh, N. Gupta, R. Gupta, and S. K. Srivastava, "Correlative analysis of long-term cosmic ray modulation with solar activity parameters," Indian Journal of Scientific Research, vol. 2, no. 4, p. 11, 2011.

[13] M. Zhao, J. Chen, Y. Liu, A. Ibrahim, X. Yan, and J. Dun, "Statistical analysis of sunspot groups and flares for solar maximum and minimum," Science Sinica Physica, Methanica \& Astronomica, vol. 44, no. 1, p. 109, 2014.

[14] P. R. Gazis, J. D. Richardson, and K. I. Paularena, "Long term periodicity in solar wind velocity during the last three solar cycles," Geophysical Research Letters, vol. 22, no. 10, pp. 1165-1168, 1995.

[15] R. Kane, "Comparison of the variations of solar indices, interplanetary plasma parameters, and cosmic ray neutron monitor intensities during 1991-2001," Journal of Geophysical Research: Space Physics, vol. 108, no. A1, 2003.

[16] E. Marsch, "Kinetic physics of the solar corona and solar wind," Living Reviews in Solar Physics, vol. 3, no. 1, p. 1, 2006.

[17] E. Marsch, "Solar wind responses to the solar activity cycle," Advances in Space Research, vol. 38, no. 5, pp. 921-930, 2006.

[18] D. McComas, M. Velli, W. S. Lewis et al., "Understanding coronal heating and solar wind acceleration: case for in situ near-sun measurements," Reviews of Geophysics, vol. 45, no. 1, 2007.

[19] T. H. Zurbuchen, "A new view of the coupling of the sun and the heliosphere," Annual Review of Astronomy and Astrophysics, vol. 45, no. 1, pp. 297-338, 2007.

[20] R. P. Kane, "Early history of cosmic rays and solar wind-some personal remembrances," Advances in Space Research, vol. 44, no. 10 , pp. 1252-1255, 2009.

[21] M. J. Owens and R. J. Forsyth, "The heliospheric magnetic field," Living Reviews in Solar Physics, vol. 10, no. 1, p. 5, 2013.

[22] K. J. Li, J. Zhang, and W. Feng, "Periodicity for $50 \mathrm{yr}$ of daily solar wind velocity," Monthly Notices of the Royal Astronomical Society, vol. 472, no. 1, pp. 289-294, 2017.

[23] R. Mishra, R. Agarwal, and S. Tiwari, "Solar cycle variation of cosmic ray intensity along with interplanetary and solar wind plasma parameters," Latvian Journal of Physics and Technical Sciences, vol. 45, no. 3, pp. 63-68, 2008.

[24] J. R. Jokipii and B. Thomas, "Effects of drift on the transport of cosmic rays. IV-modulation by a wavy interplanetary current sheet," The Astrophysical Journal, vol. 243, p. 1115, 1981.

[25] H. V. Cane, G. Wibberenz, I. G. Richardson, and T. T. Von Rosenvinge, "Cosmic ray modulation and the solar magnetic field," Geophysical Research Letters, vol. 26, no. 5, pp. 565-568, 1999.

[26] H. S. Ahluwalia, "On galactic cosmic ray flux decrease near solar minima and IMF intensity," Geophysical Research Letters, vol. 27, no. 11, pp. 1603-1606, 2000.

[27] A. Hempelmann and W. Weber, "Correlation between the sunspot number, the total solar irradiance, and the terrestrial insolation," Solar Physics, vol. 277, no. 2, pp. 417-430, 2012.

[28] X. L. Yan, L. H. Deng, Z. Q. Qu, and C. L. Xu, "The phase relation between sunspot numbers and soft X-ray flares," Astrophysics and Space Science, vol. 333, no. 1, pp. 11-16, 2011.

[29] J. Pérez-Peraza, V. Velasco, I. Y. Libin, and K. Yudakhin, "Thirty-year periodicity of cosmic rays," Advances in astronomy, vol. 2012, Article ID 691408, 11 pages, 2012. 
[30] K. J. Li, J. Zhanng, and W. Feng, "A statistical analysis of 50 years of daily solar wind velocity data," The Astronomical Journal, vol. 151, no. 5, p. 128, 2016.

[31] J. Oloketuyi, Y. Liu, and M. Zhao, "The periodic and temporal behaviors of solar X-ray flares in solar cycles 23 and 24," The Astrophysical Journal, vol. 874, no. 1, p. 20, 2019.

[32] M. Hagino, T. Sakurai, and A. Miyazawa, "Phase relationship between the activity cycles of sunspots and polar faculae," The Solar-B Mission and the Forefront of Solar Physics, vol. 157, 2004.

[33] A. Grinsted, J. C. Moore, and S. Jevrejeva, "Application of the cross wavelet transform and wavelet coherence to geophysical time series," Nonlinear Processes in Geophysics, vol. 11, no. 5/6, pp. 561-566, 2004.

[34] Y. P. Singh, "Prominent short-, mid-, and long-term periodicities in solar and geomagnetic activity: wavelet analysis," Planetary and Space Science, vol. 96, pp. 120-124, 2014.

[35] R. Badruddin, J. L. Ballester, and F. Baudin, "Emergence of magnetic flux on the sun as the cause of a 158-day periodicity in sunspot areas," Nature, vol. 394, no. 6693, pp. 552-553, 1998.

[36] C. Torrence and G. P. Compo, "A practical guide to wavelet analysis," Bulletin of the American Meteorological Society, vol. 79, no. 1, pp. 61-78, 1998.

[37] M. Fligge, S. Solanki, and J. Beer, "Determination of solar cycle length variations using the continuous wavelet transform," Astronomy and Astrophysics, vol. 346, p. 313, 1999.

[38] J. Polygiannakis, P. Preka-Papadema, and X. Moussas, "On signal-noise decomposition of time-series using the continuous wavelet transform: application to sunspot index," Monthly Notices of the Royal Astronomical Society, vol. 343, no. 3, pp. 725-734, 2003.

[39] V. Velasco, B. Mendoza, and J. Valdes-Galicia, "The 120-yrs solar cycle of the cosmogenic isotopes," International Cosmic Ray Conference, vol. 553, 2008.

[40] N. Marwan, M. Thiel, and N. R. Nowaczyk, "Cross recurrence plot based synchronization of time series," 2002, https://arxiv. org/abs/physics/0201062.

[41] R. Haddadi, E. Abdelmounim, M. El Hanine, and A. Belaguid, "Discrete wavelet transform based algorithm for recognition of QRS complexes," in Proceedings of the International Conference on Multimedia Computing and Systems (ICMCS), vol. 375, IEEE, Marrakesh, Morocco, April 2014.

[42] N. Marwan and J. Kurths, "Nonlinear analysis of bivariate data with cross recurrence plots," Physics Letters A, vol. 302, no. 5-6, p. 299, 2002.

[43] D. Maraun and J. Kurths, "Cross wavelet analysis: significance testing and pitfalls," Nonlinear Processes in Geophysics, vol. 11, no. 4, pp. 505-514, 2004.

[44] J. G. Luhmann, G. Petrie, and P. Riley, "Solar origins of solar wind properties during the cycle 23 solar minimum and rising phase of cycle 24," Journal of Advanced Research, vol. 4, no. 3, pp. 221-228, 2013.

[45] B. L. Burkholder, A. Otto, P. A. Delamere, and J. E. Borovsky, "Magnetic connectivity in the corona as a source of structure in the solar wind," Journal of Geophysical Research: Space Physics, vol. 124, no. 1, pp. 32-49, 2019.

[46] S. E. Forbush, "World-wide cosmic ray variations, 1937-1952," Journal of Geophysical Research, vol. 59, no. 4, pp. 525-542, 1954.

[47] S. E. Forbush, "Cosmic-ray intensity variations during two solar cycles," Journal of Geophysical Research, vol. 63, no. 4, pp. 651-669, 1958.
[48] M. Mishra, "Solar activity and cosmic ray intensity variation," in Proceedings of the International Cosmic Ray Conference, p. 159, Pune, India, August 2005.

[49] B. Tiwari, B. Ghormare, P. Shrivastava, and D. Tiwari, "Modulation in cosmic ray during the declining and minimum solar activity period of solar cycle 23," Research Journal of Physical Sciences, vol. 2, no. 2, p. 9, 2014.

[50] B. A. Emery, I. G. Richardson, D. S. Evans, and F. J. Rich, "Solar wind structure sources and periodicities of auroral electron power over three solar cycles," Journal of Atmospheric and Solar-Terrestrial Physics, vol. 71, no. 10-11, pp. 1157-1175, 2009.

[51] Y. P. Singh and S. Gautam, "Temporal variations of short- and mid-term periodicities in solar wind parameters and cosmic ray intensity," Journal of Atmospheric and Solar-Terrestrial Physics, vol. 89, pp. 48-53, 2012.

[52] P. Badruddin, B. Heber, A. Kopp et al., "Observations of recurrent cosmic ray decreases during solar cycles 22 and 23," Annales Geophysicae: Atmospheres, Hydrospheres and Space Sciences, vol. 3127, 2008.

[53] D. Lario and E. Roelof, "Energetic particles during the first and third ulysses southern high-latitude excursions: probing global corotating interaction region structure beyond $5 \mathrm{AU}$," Journal of Geophysical Research: Space Physics, vol. 112, no. A9, 2007.

[54] B. Joshi, P. Pant, and P. K. Manoharan, "Periodicities in sunspot activity during solar cycle 23," Astronomy \& Astrophysics, vol. 452, no. 2, pp. 647-650, 2006.

[55] B. Mendoza and V. M. Velasco-Herrera, "On mid-term periodicities in sunspot groups and flare index," Solar Physics, vol. 271, no. 1-2, pp. 169-182, 2011.

[56] P. Chowdhury, M. Khan, and P. C. Ray, "Intermediate-term periodicities in sunspot areas during solar cycles 22 and 23," Monthly Notices of the Royal Astronomical Society, vol. 392, no. 3, pp. 1159-1180, 2009.

[57] Z.-Q. Yin, Y.-B. Han, L.-H. Ma, G.-M. Le, and Y.-G. Han, "Short-term period variation of relative sunspot numbers," Chinese Journal of Astronomy and Astrophysics, vol. 7, no. 6, pp. 823-830, 2007.

[58] A. Kilcik, V. Yurchyshyn, F. Clette, A. Ozguc, and J.-P. Rozelot, "Active latitude oscillations observed on the sun,” Solar Physics, vol. 291, no. 4, pp. 1077-1087, 2016.

[59] Y. Singh, "Study of the solar rotational period and its harmonics in solar activity, interplanetary, geomagnetic, and cosmic ray intensity indicators during solar polarity reversal periods," Solar Physics, vol. 294, no. 3, p. 27, 2019.

[60] M. Tsichla, M. Gerontidou, and H. Mavromichalaki, "Spectral analysis of solar and geomagnetic parameters in relation to cosmic-ray intensity for the time period 1965-2018," Solar Physics, vol. 294, no. 1, p. 15, 2019.

[61] R. Donner and M. Thiel, "Scale-resolved phase coherence analysis of hemispheric sunspot activity: a new look at the north-south asymmetry," Astronomy \& Astrophysics, vol. 475, no. 3, pp. L33-L36, 2007.

[62] L. H. Deng, Z. Q. Qu, K. R. Wang, and X. B. Li, "Phase asynchrony between coronal index and sunspot numbers," Advances in Space Research, vol. 50, no. 10, pp. 1425-1433, 2012.

[63] M. Carbonell, R. Oliver, and J. Ballester, "On the asymmetry of solar activity," Astronomy and Astrophysics, vol. 274, p. 497, 1993. 